\title{
métodos modernos de excavación de galerías y túneles
}

\section{sinopsis}

GEORGES VIÉ, ingeniero de minas

El avance extraordinario registrado en los últimos años en el campo de las obras públicas se debe, fundamentalmen. te, al empleo masivo de maquinaria auxiliar en cualquier tajo de una obra.

Como muestra evidente tenemos el ejemplo de las excavaciones subterráneas, en las que la adopción progresiva de elementos auxiliares ha permitido rendimientos y economías insospechados.

Entre los elementos auxiliares de reciente introducción figuran los JUMBOS, aparatos móviles autopropulsados, o no, que sirven de soporte a los martillos perforadores.

Al no exigir del obrero un esfuerzo físico directo, se ha podido llegar a pesos notables en los martillos, con velocidades de perforación elevadas.

Estas máquinas presentan una serie de características directamente relacionadas con la naturaleza de la obra y la forma de la sección a excavar, pero sus características esenciales son siempre las mismas.

El presente artículo describe algunas variantes de estas máquinas, que se han empleado, con pleno éxito, en diversas obras de Francia.

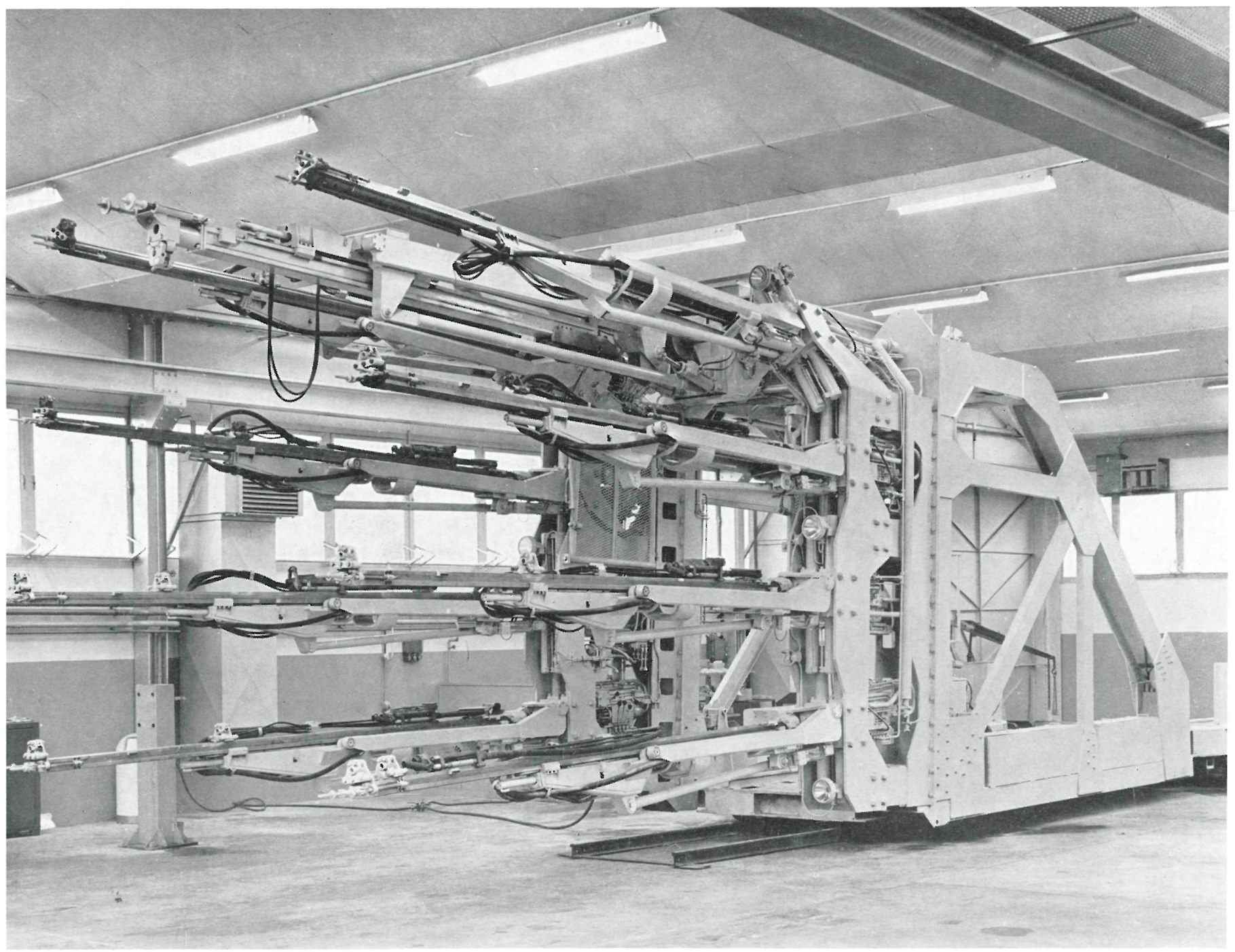




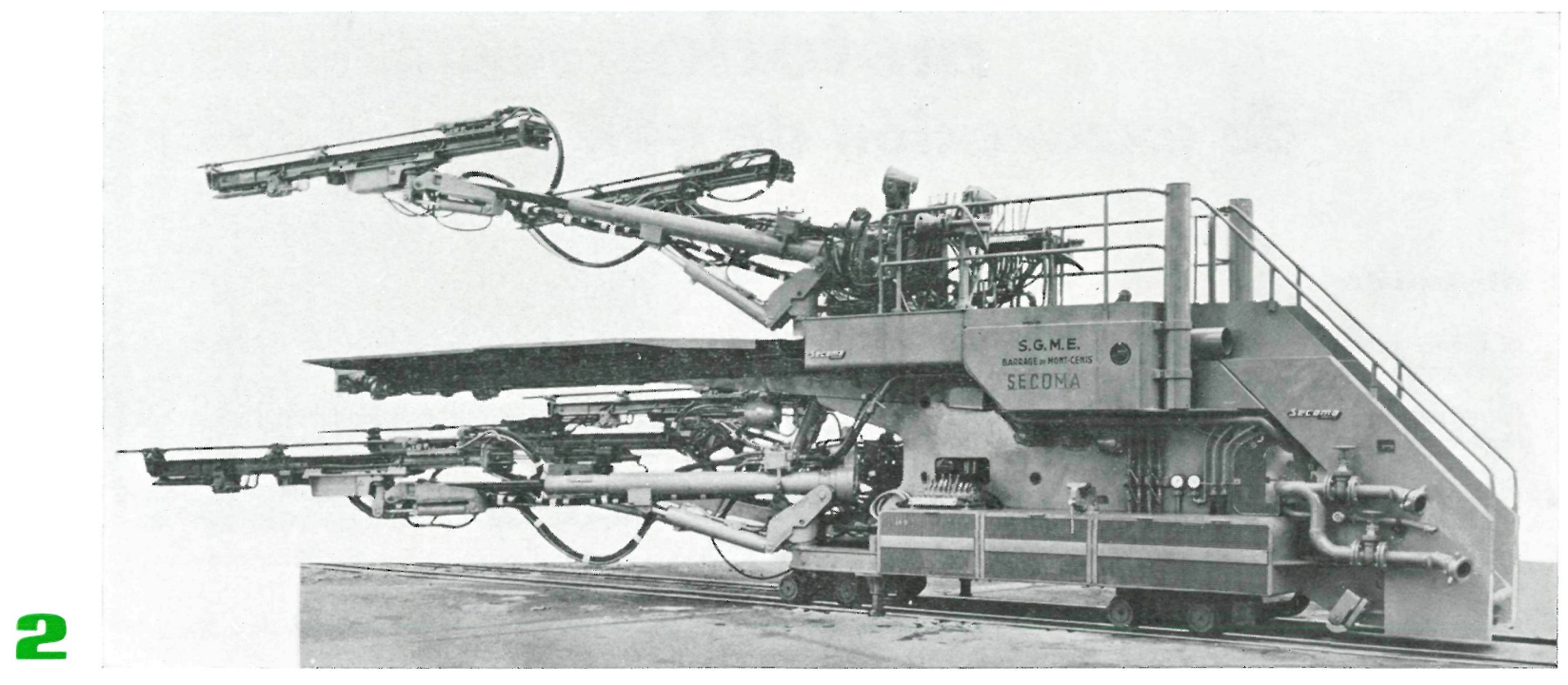

Foto: MARCEL LOMBARD (LyOn)

El progreso que ha experimentado en los últimos años la excavación de galerías, se ha debido en gran parte a los dispositivos denominados JUMBOS.

Los primeros modelos que se utilizaron eran unas plataformas móviles con brazos articulados, sobre los que se montaban los martillos perforadores de aire comprimido. El desplazamiento de estas plataformas se hacía con elementos tractores independientes. Se ha evolucionado rápidamente hacia plataformas autopropulsadas de gran autonomía.

La tendencia a aumentar el peso de los martillos perforadores no tiene más limitación que la que impone el tamaño del material excavado, obteniéndose espectaculares velocidades de avance con cualquier tipo de sección excavada. Los JUMBOS pueden desplazarse sobre vía férrea, sobre neumáticos o sobre orugas, utilizando como fuentes de energía la electricidad o el aire comprimido. La perforación puede realizarse por percusión, rotación o por un procedimiento combinado de ambos métodos.

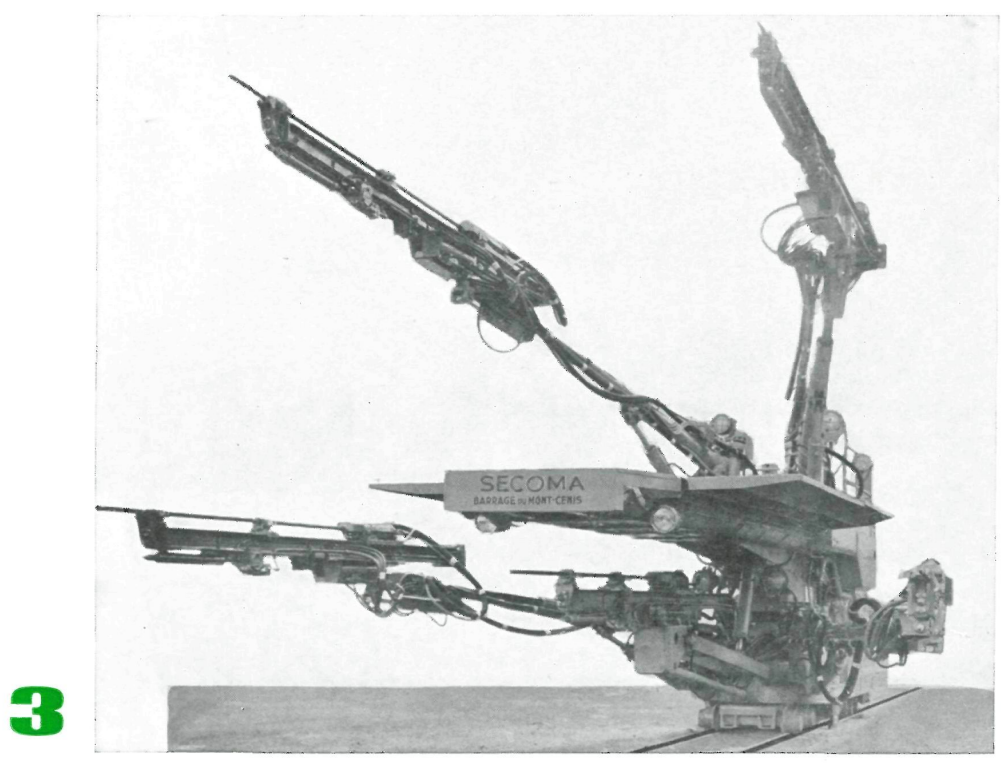

Estas máquinas pueden llevar un número de brazos, función de la sección a excavar y de los rendimientos previstos. Han sido muy utilizadas en Europa y América, encontrando gran aplicación en explotaciones mineras y trabajos de obras públicas. Existen diversidad de modelos que se adaptan lo mejor posible al uso a que se les destina.

Su introducción en el campo de las obras públicas ha mejorado notablemente los rendimientos con los trabajos de perforación de galerías y túneles, siendo rara la obra en la que no se emplean.

La maniobrabilidad de los JUMBOS permite realizar voladuras en un tiempo mínimo, con una economía de mano de obra del $50 \%$ aproximadamente. La disposición de los taladros se ajusta a procedimientos diversos. El cuele ca- 
nadiense consiste en perforar uno o varios taladros centrales de gran diámetro que se dejan sin cargar, permitiendo la expansión del material en sus proximidades.

Los JUMBOS tipo SECOMA se han empleado, con éxito, en las excavaciones de galerías en los aprovechamientos de Saint Georges de Commiers, Curbans, Mont-Cenis, Canal de Provenza, etc., pertenecientes a Electricité de France.

En los frentes de ataque de la galería de Suffet (Salto de Mont-Cenis) se han empleado JUMBOS SECOMA de cinco brazos con desplazamiento sobre vía férrea (figs. 2 y 3). Cada JUMBO está formado por cuatro brazos con mando hidráulico (JTH), con martillo MEUDON MP 50, sobre avanzadores de 3,20 m de recorrido útil y un brazo central con martillo $\mathrm{D} 475$, de $3,20 \mathrm{~m}$ de recorrido útil.

La sección de ataque es de $23 \mathrm{~m}^{2}$ y la voladura exigió la perforación de:

cincuenta taladros de $37 \mathrm{~mm}$ de diámetro cargados con explosivo tolamite;

cinco taladros de $48 \mathrm{~mm}$ de diámetro cargados con grisú-dinamita;

tres taladros de expansión paralelos a $102 \mathrm{~mm}$ de diámetro.

El ciclo medio de voladura comprendía los tiempos siguientes:

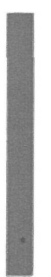

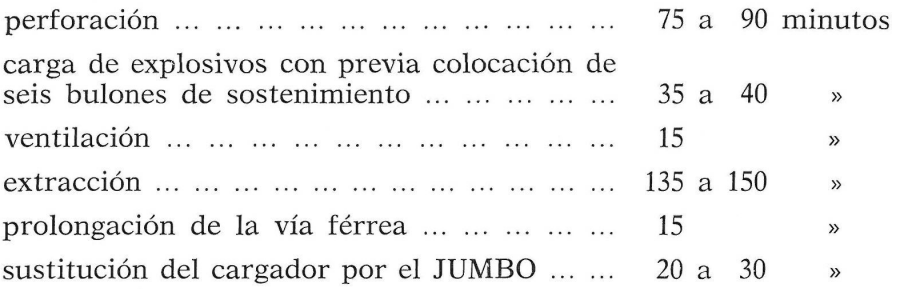

La duración total de un ciclo ha variado entre $4 \mathrm{~h} 55$ min y 5 h 40 minutos. El avance medio en cada frente de ataque, comprendido el bulonado (bulones ANCRAL de expansión de 2,5 m), ha sido de $260 \mathrm{~m}$ por mes.

En febrero de 1966 el avance fue de $260 \mathrm{~m}$, con dos récord de $40 \mathrm{~m}$ en tres días y $78 \mathrm{~m}$ en seis días.

La aspiración se realizó con tubería de $800 \mathrm{~mm}$ de diámetro, con su boca aspirante situada a $20 \mathrm{~m}$ del frente de ataque.

La mano de obra empleada en cada turno era de ocho hombres; cuatro mineros, un mecánico, un conductor de tractor, un cargador y un encargado de turno. En un turno por la mañana de cinco obreros complementarios efectuaban el alargamiento de tuberías de aire comprimido, canales de ventilación y vías férreas.

Cada $600 \mathrm{~m}$ se disponían apartaderos para el cruce del JUMBO con los elementos de evacuación.

El consumo medio de cada martillo era de $9 \mathrm{~m}^{3} / \mathrm{s}$, y el de la sonda de gran diámetro, de $19 \mathrm{~m}^{3} / \mathrm{minuto}$. El consumo de explosivo fue del orden de $2.500 \mathrm{~g} / \mathrm{m}^{3}$. La potencia de los compresores era de $500 \mathrm{CV}$, con una unidad suplementaria de $90 \mathrm{CV}$ durante el invierno.

Solamente hombres especializados en el manejo de los mandos hidroneumáticos de los brazos del JUMBO pueden obtener el máximo beneficio de este ingenio de perforación. El dispositivo de mandos está formado por ocho palancas de accionamiento que exigen del operador una técnica y destreza parecidas a la de la propia máquina.

La extracción de los productos excavados se realizó de la forma siguiente:

Una pala EIMCO-40 cargaba los escombros a un transportador acumulador (SOTIM-CAR) (fig. 4), que, a su vez, carga las vagonetas ARBEL, de $3,6 \mathrm{~m}^{3}$ de capacidad y descarga lateral. El esquema de trabajo es el siguiente:

Cuatro carriles paralelos equidistantes determinan tres vías: la pala EIMCO-40 está colocada en la vía central; el SOTIM-CAR, en la vía derecha, y las vagonetas vacías, en la vía izquierda. El SOTIM-CAR dis- 
pone de un cable arrollado sobre un torno, con el que se colocan las vagonetas en posición favorable para ser cargadas. Cuando la vagoneta está llena, la envía hacia el tren de vagonetas dispuesto para la extracción. El cable es desatado y se fija de nuevo en una vagoneta vacía.

Este sistema no deja prácticamente tiempos muertos. El personal utilizado está compuesto por:

Un obrero que asegura la limpieza del frente, un conductor de la pala EIMCO, un obrero para manejar el torno del SOTIM-CAR, un obrero para la eslinga de las vagonetas, y otro obrero para el riego.

La duración total de las operaciones (evacuación de los gases del tiro, colocación y alargamiento de la vía) es de 190 minutos.

La duración de un cuelo completo es aproximadamente de 6 horas, obteniéndose un avance de cada frente de $9 \mathrm{~m}$ por día.

Posteriormente se han introducido soluciones elegantes y prácticas para resolver el problema de extracción de escombros, suprimiendo las sucesivas maniobras de ida y vuelta del JUMBO y la pala mecánica. La utilización del cambio canadiense permite el cruce de ambas máquinas entre sí o con las vagonetas en las proximidades del frente. Para ello es necesario una anchura mínima de la sección excavada o bien un ensanchamiento localizado, que sirve de apartadero. Los nuevos dispositivos empleados son: el JUMBO-cargador, el JUMBO-pórtico, y el CHERRY-picker (puente-grúa para el apartado de vagonetas).

Se hacía sentir la necesidad de agrupar sobre un aparato único el conjunto de las operaciones que entraña la excavación profunda. Las dificultades técnicas y mecánicas por resolver retardaban su introducción en el terreno práctico. El JUMBO-cargador representó un notable avance en el perfeccionamiento de estas máquinas.

El JUMBO-cargador queda en el frente durante todo el ciclo, exceptuando la voladura. La sección óptima de excavación oscila entre 9 y $20 \mathrm{~m}^{2}$. Para secciones mayores se han aportado otras soluciones, principalmente el JUMBO-pórtico, el cual permite a los dispositivos de carga y extracción pasar bajo el dispositivo de perforación.

\section{El JUMBO-cargador comprende:}

Bastidor con los mecanismos de traslación, los brazos de perforación montados en su parte delantera, y el dispositivo de carga por arrastre y extracción de los escombros, que atraviesan la máquina en sentido anterior a posterior.

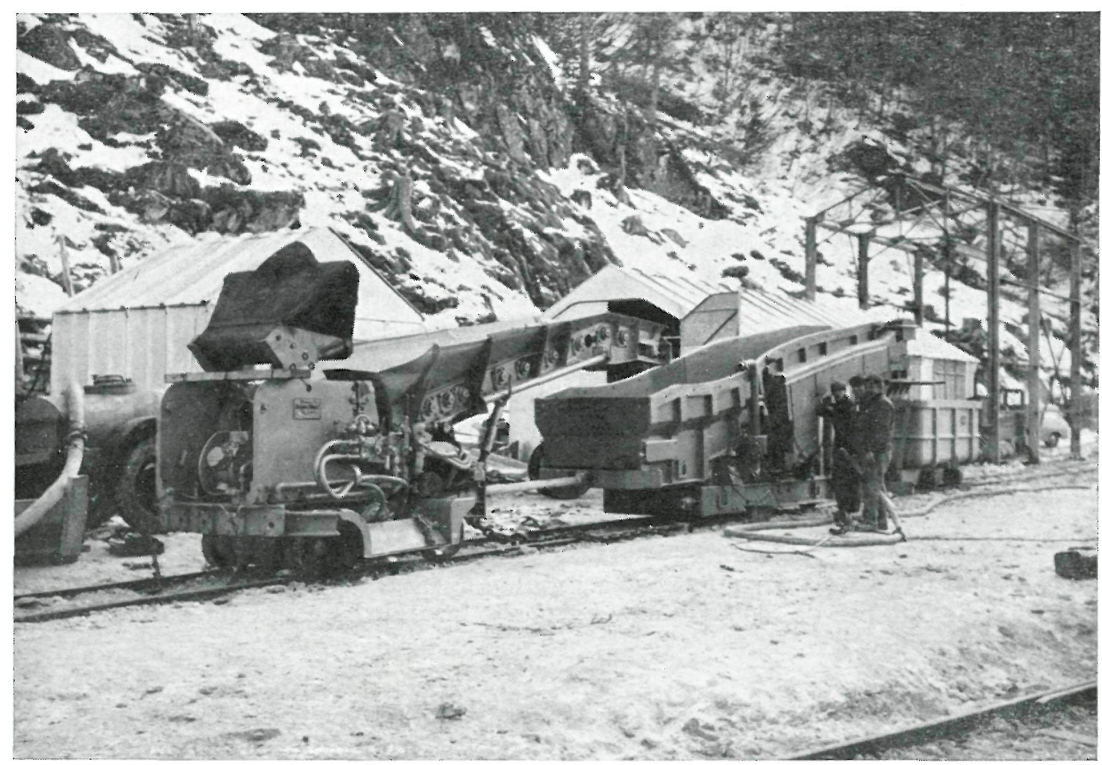






En la galería de Peyrolles (Concors), incluida en el Plan de Construcción del Canal de Provenza, se ha empleado un JUMBO-cargador de tres brazos, preparado para un frente de ataque de $10,5 \mathrm{~m}^{2}$. Cada uno de los brazos lleva una barrena de perforación rotativa de gran presión, con carrera útil de 2,20 metros (fig. 5).

La perforación rotativa en las calizas cretáceas es más rápida y económica que la perforación por percusión. No obstante, se puede sustituir un tipo de martillo por otro cuando así lo aconseja la dureza de la roca.

La carga de los escombros de cada voladura se ha realizado por arrastre. El JUMBO-cargador dispone de un torno con cable arrollado para el manejo de una pala excavadora. La polea del cable va sujeta al extremo de la deslizadera soporte del brazo central de perforación (fig. 6).

Los productos excavados son canalizados hacia las cintas extractoras por medio de una placa articulada que va en la parte delantera del JUMBO. Esta placa va provista de gatos hidráulicos que le permiten adaptarse al terreno, dejándole nivelado a medida que la máquina avanza hacia el frente del ataque.

La cinta metálica del JUMBO puede cargar directamente las vagonetas o por intermedio de una cinta transportadora (fig. 7). Durante la carga de escombros, cada uno de los dos brazos laterales perfora cuatro taladros de $2,20 \mathrm{~m}$ de longitud.

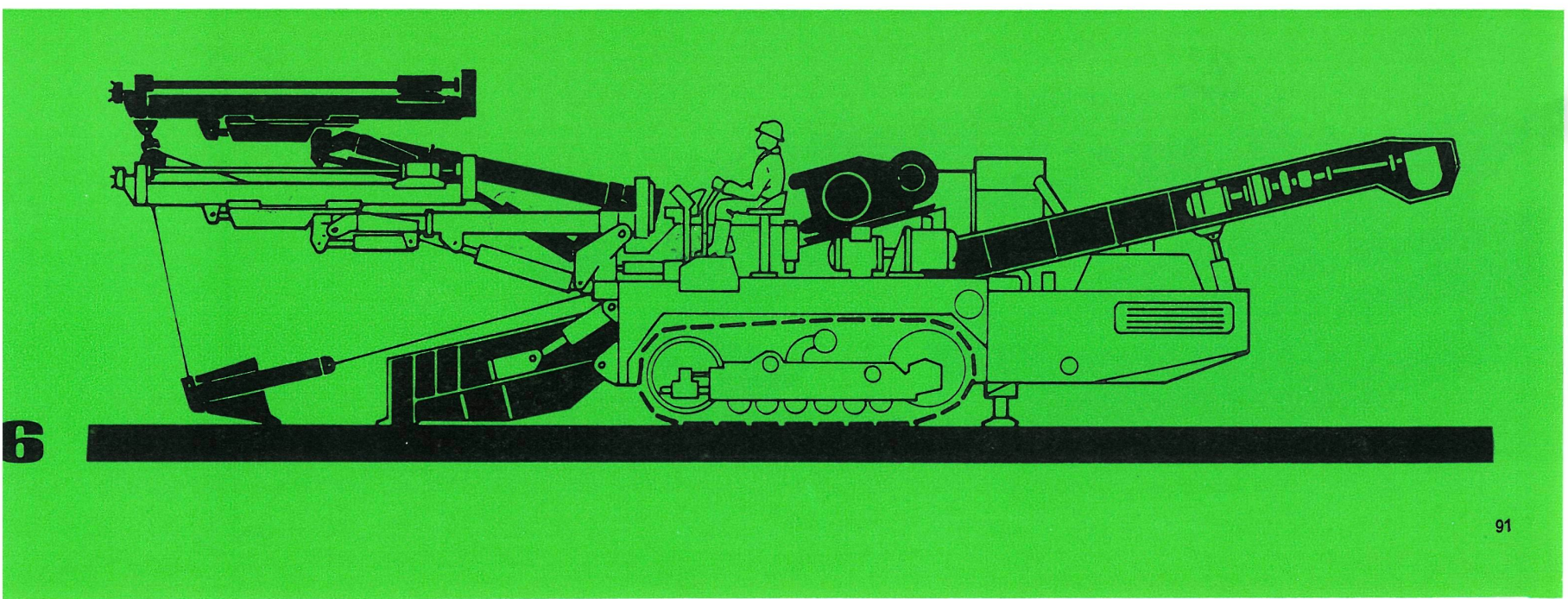




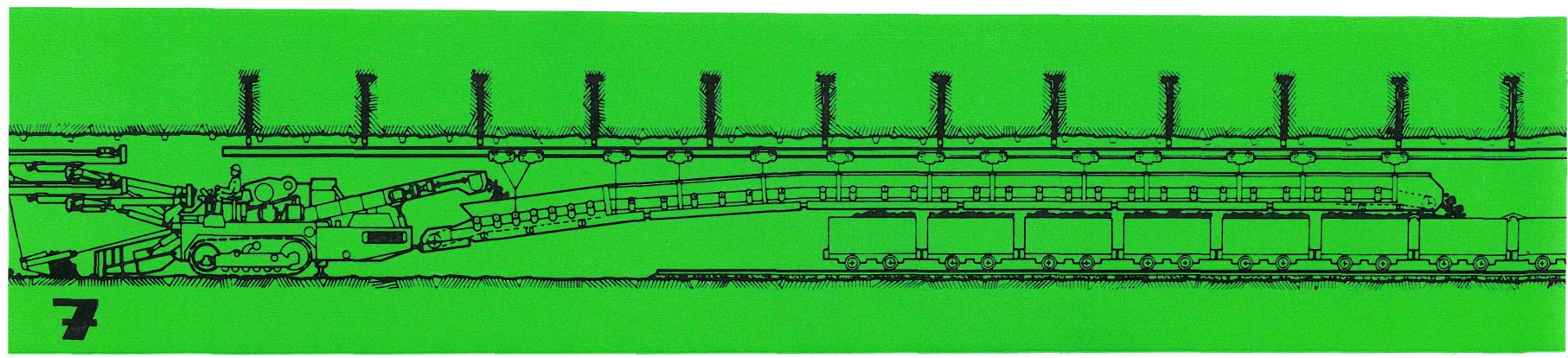

Los avances diarios han oscilado entre $16,3 \mathrm{~m}$ y $18 \mathrm{~m}$. En sección de $10,5 \mathrm{~m}^{2}$ ha sido fácil hacer nueve ciclos completos por cada día, con duración media entre 2 h 15 min y 2 h 30 minutos.

El sistema (Ahros-Chambery) aporta a la excavación en roca una solución de concepción distinta a las anteriores.

El dispositivo lo forman cinco pórticos articulados de $15 \mathrm{~m}$ de longitud, unidos por largueros de 6,5 $\mathrm{m}$ que forman pasarelas a ambos lados.

El canal de ventilación, los conductos de agua y los cables eléctricos están colocados sobre las pasarelas de una manera fija. Estas canalizaciones enlazan en la parte posterior del tren con las canalizaciones fijas colocadas sobre el paramento de la galería.

El pórtico posterior está equipado con plataforma de trabajo y mandos de accionamiento. Contiene, además, el dispositivo de llenado de vagones, con mando eléctrico.

Los tres pórticos intermedios soportan las cintas transportadoras. El tren se desplaza sobre vía férrea de $2,80 \mathrm{~m}$ de ancho, con elementos tractores eléctricos de 380 voltios.

La excavación de galerías en zona urbana presenta otro tipo de problemas, como son la imposibilidad de realizar voladuras, para no perturbar las construcciones próximas, y las dificultades de evacuación de los productos excavados.

Para excavar una galería bajo el Sena, en París, de $12 \mathrm{~m}^{2}$ de sección, se ha construido un JUMBO excavador-cargador, tipo SECOMA, cuyos brazos van provistos de martillos rompe-rocas MEUDON BR. 50 ó BR. 70 (fig. 8).

El terreno a excavar está formado por yesos, y su evacuación se realiza por pozos con boca de salida en el centro de una calle de gran circulación. Por otra parte, el trabajo se efectúa en un solo turno durante el día, por lo que el rendimiento de la máquina es del $50 \%$ de sus posibilidades reales.

Durante el trabajo de excavación la pala de que va provista la máquina carga la cinta metálica incorporada también al JUMBO, de donde se distribuyen los productos excavados a unos cubos de 900 litros de capacidad, situados de dos en dos sobre unas vagonetas. Estos cubos se extraen a la superficie por medio de una grúa de 2 toneladas.

El JUMBO consume un total de $16 \mathrm{~m}^{3} / \mathrm{min}$ de aire comprimido y va montado sobre orugas TP 6 Continental. La capacidad de la pala cargadora es de 250 litros, y el rendimiento teórico de la cinta metálica, $170 \mathrm{~m}^{3} /$ hora.

Traducido y adaptado por J. A. Piedra.

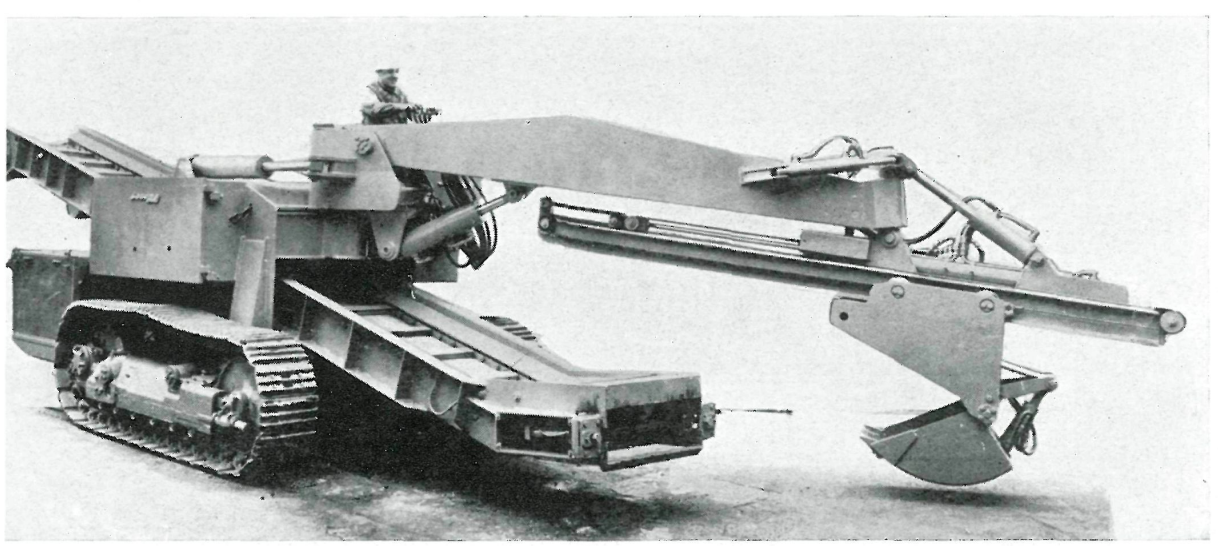




\section{Méthodes modernes de creusement des gulemies et tummels} Georges Vié, ingénieur des mines.

Les progrès extraordinaires réalisés ces dernières années dans le domaine des travaux publics sont dus principalement à l'emploi massif d'engins auxiliaires pour tous les types de travaux.

Ces progrès sont mis en évidence par l'exemple des perforations souterraines, où l'adoption progressive des éléments auxiliaires a permis d'obtenir des performances et des économies inimaginables.

Parmi les éléments auxiliaires récemment mis en usage figurent les «jumbos», engins mobiles automoteurs ou non, qui servent de support aux marteaux perforateurs.

L'effort physique directement réalisé par le mineur n'étant plus nécessaire, il a été possible d'envisager des poids considérables pour les marteaux atteignant des vitesses de perforation élevées.

Ces engins présentent une série de caractéristiques directement liées à la nature de l'ouvrage et à la forme de la section à perforer, mais leurs caractéristiques essentielles sont toujours les mêmes.

Cet article décrit quelques types de ces engins utilisés en France avec plein succès en divers ouvrages.

\section{Modem methods to excavate gallemies and tummels}

Georges Vié, mining engineer

The outstanding progress in recent years in the field of public works is basically due to the massive use of machinery at any working site.

An obvious example is that of underground excavation, where the progressive use of more advanced machinery has made it possible to attain exceptional performances and reductions in cost.

Among the equipment items that have been recently introduced mention should be made of the Jumbos, which are selfpropelled mobile devices, and serve as supporting platforms for the drilling hammers.

As the operators are no longer obliged to handle directly the drilling machines, these can now be made much heavier and can attain much higher drilling rates.

These devices have a number of features that are directly related to the nature of the work and the cross section to be excavated, but their general design is common to all of them.

This article describes some variants of these Jumbos, which have been successfully employed at various working sites in France.

\section{Moderne Methoden fuer das Ausbaggern won Stollem und Tummels}

Georges Vié, Bergingenieur

Der grosse Fortschritt, der in den letzten Jahren auf dem Gebiete der öffentlichen Bauten erzielt wurde, ist hauptsächlich dem massiven Einsatz von Hilfsmaschinen bei irgendwelcher Arbeit auf einer Baustelle zuzuschreiben.

Als offensichtliches Beispiel seien die unterirdischen Ausbaggerungen genannt, bei denen der progressive Einsatz von Hilfselementen ungeahnte Leistungen und Einsparungen ermöglicht hat.

Unter den neuen Hilfselementen befinden sich die JUMBOS. Es handelt sich dabei um bewegliche Apparate, mit oder ohne Selbstantrieb, die als Träger der Bohrhämmer dienen.

Dadurch, dass der Arbeiter keine direkte körperliche Anstrengung aufzubringen hat, konnte man beträchtliche Hammerge wichte mit hohen Bohrgeschwindigkeiten erreichen.

Diese Maschinen haben eine Reihe von Merkmalen, die in direktem Zusammenhang mit der Art der Baustelle und der Form des auzubaggernden Querschnitts stehen, doch sind deren Hauptmerkmale stets dieselben.

Der vorliegende Artikel beschreibt einige Varianten dieser Maschinen, die auf verschiedenen Baustellen Frankreich mit vollem Erfolg zum Einsatz gekommen sind. 